\title{
Is it time for a moratorium on the use of benzalkonium chloride in eye drops?
}

\author{
Luca D’andrea ${ }^{1}$, Marina Montemagni ${ }^{1}$, Giuseppe Celenza ${ }^{2}$, Roberto Iorio $^{2}$, and Ciro \\ Costagliola ${ }^{1}$ \\ ${ }^{1}$ University of Naples Federico II \\ ${ }^{2}$ University of Aquila
}

February 1, 2022

\begin{abstract}
Benzalkonium chloride (BAC) is a quaternary ammonium compound commonly employed as a preservative in several multidose eye drops. Its use is associated with several adverse events, particularly when used chronically. Topically instilled BAC acts not only on superficial structures but also reaches deeper tissues (trabecular meshwork, optic nerve) and can be deleterious in patient under chronic treatment. In glaucomatous patients, BAC is the primary cause of ocular surface disease (OSD), and it can lead to significant morbidity, influence treatment compliance, quality of life, and surgical outcomes. Clinical and experimental evidence demonstrates that BAC causes instability of the tear layer, loss of goblet cells, apoptosis, subclinical neuroinflammation, and antibiotic resistance. Considering the validity of the alternative formulations (alternative preservatives or preservative-free formulations) it is unreasonable to persist in using such toxic compounds and, perhaps it is time for a moratorium on the use of BAC in eye drops.
\end{abstract}

Title: Is it time for a moratorium on the use of benzalkonium chloride in eye drops?

D'andrea $\mathrm{L}^{1}$, Montemagni $\mathrm{M}^{2}$, Celenza $\mathrm{G}^{3}$, Iorio $\mathbf{R}^{3}$, Costagliola $\mathrm{C}^{2}$.

1. Department of Public Health, University of Naples Federico II, 80131, Naples, Italy

2. Department of Neurosciences, Reproductive and Dentistry Sciences, University of Naples Federico II, 80131, Naples, Italy

3. Department of Biotechnological and Applied Clinical Sciences, University of Aquila, 67100, Aquila, Italy

Corresponding author : Marina Montemagni, MD

Department of Neurosciences, Reproductive and Dentistry Sciences, University of Naples Federico II, Naples, Italy. Via Sergio Pansini 15, 80131, Naples, Italy

Email:marinamontemagni91@gmail.com

Phone: +393200294414

ORCID 0000-0002-7981-3383

The authors declare no conflict of interest.

Keywords : Benzalkonium chloride, BAC, OSD, toxicity, glaucoma, neuroinflammation.

Word count: 1001

Figure count: 1 


\section{Commentary}

Benzalkonium chloride (BAC) is a quaternary ammonium compound introduced in 1935 and widely used as a skin and surface disinfectant and preservative in many household cleaning and personal care products. Due to its antimicrobial activity against many common pathogens, BAC is also employed as a preservative in several multidose eyedrops at concentrations varying from $0.004 \%$ to $0.02 \%$. The success and longevity of $\mathrm{BAC}$ are attributable to its undeniable antibacterial efficacy and, not least, its low production cost ${ }^{1}$.

As a cationic surfactant, it has an amphipathic nature, and it expresses the antimicrobial activity denaturing proteins and disrupting cytoplasmatic membranes. BAC is also used as an excipient to stabilize poor watersoluble drugs and as a penetration enhancer of active compounds through ocular membranes. Indeed, it is believed that BAC can solubilize the intercellular junctions in corneal epithelium, enhancing drug delivery 2 .

Although anecdotal reports have been circulating in the last years about the adjuvant effect of BAC as an enhancer of the prostaglandin analogues efficacy, recent studies have demonstrated that BAC does not change the pharmacokinetics of the active ingredients. In fact, many studies on glaucomatous patients show a similar intraocular pressure (IOP) reduction in patients receiving BAC-containing and preservative-free formulations. Pellinen and Lokkilla compared corneal penetration of $0.01 \%$ BAC preserved and preservative-free tafluprost in rabbit aqueous humor after topical administration. No statistically significant difference in the mean concentration over time was recorded (the maximum drug concentration was $4.50 \mathrm{ng} / \mathrm{mL}$ for preservative-free tafluprost and $3.99 \mathrm{ng} / \mathrm{mL}$ for $0.01 \%$ BAC-preserved tafluprost, and the time to maximum drug concentration was $45 \mathrm{~min}$ for both) ${ }^{3}$. This finding demonstrated that BAC is far from an ideal penetration enhancer.

Eye drops containing BAC are associated with some adverse events, particularly when used chronically, like in glaucomatous patients. The European Medicine Agency (EMA) suggests to avoid preservatives in glaucomatous patients, especially in whose with pre-existing Ocular Surface Disease (OSD) or in those developing dry eye or ocular irritation over time.

The development of OSD in glaucomatous patients depends primarily on BAC, which affects lipid layer and tears film stability. Moreover, it decreases tear break-up time (TBUT), induces apoptosis of conjunctival cells with a breakdown of corneal epithelial junctions and loss of superficial epithelial cells. It is not surprising that patients treated with IOP-lowering eye drops show a higher percentage of OSD compared to the general elderly population (59\% vs 15\%). A positive correlation with the number of BAC-preserved eyes and drops used was observed ${ }^{4}$. OSD causes significant morbidity and influences treatment compliance, quality of life, and surgical outcomes ${ }^{5}$. On the other hand, it is not surprising that topical adverse effects represent a source of poor adherence and a common reason for drop-out in clinical situations and clinical trials, representing an important barrier to the effective management of $\mathrm{IOP}^{6}$. Long-term use of topical eye drops rich in preservatives can modify eye surfaces, compromising the success rate of trabeculectomy. In most cases, the failure of glaucoma surgery originates from a fibroblastic conjunctival response to inflammation at the bleb level, which occurs in the early postoperative months. A significant relationship between the number of drugs used, duration of treatment, inflammatory cell and fibroblast infiltration in the conjunctiva, and the risk of failure of filtering surgery has also been found ${ }^{5}$.

Topically instilled BAC acts not only on superficial structures but also reaches deeper tissues, such as trabecular meshwork and optic nerve areas, with a greater impact in case of long exposure (inflammatory cell infiltration and Müller glial cell activation), as demonstrated by Brignole-Baudouin et al. though the use of mass spectrometry imaging and immunological analyses ${ }^{7}$. Thus, BAC exposure could worsen glaucoma and, through its capacity to accumulate in the trabecular meshwork, can cause changes similar to those found in the ocular outflow pathway of glaucomatous patients. Detrimental BAC's effects could result in progressive loss of efficacy of IOP-lowering compounds. Moreover, the presence of this preservative along the optic nerve could be a sight-threatening issue: BAC side effects could account for the percentage of glaucomatous patients whose disease progress despite a good IOP control ${ }^{7}$.

Stevens et al. showed that short-term BAC administration induces inflammation in the anterior segment ${ }^{8}$. 
When a pro-inflammatory stimulus arises during injury or disease, astrocytes, Müller cells, and microglia become activated and produce cytokines and chemokines. This recruits blood-derived immune cells to the retina, which causes amplification of the inflammatory response in the retina. Thus, glaucomatous insult or elevation of IOP triggers the responses of microglia, astrocytes, and Müller cells to participate in the process of neuroinflammation. Glaucomatous patients present inflammatory dysfunction of the retinal ganglion cell layer (RGC) and the optic nerve head. BAC enhances inflammatory response both at the level of the ocular surface and along all the visual pathways ${ }^{7}$. Mitochondrial oxidative stress has been reported to promote OSD. In addition, RGC is a highly vulnerable neuronal cell type with reference to mitochondrial dysfunction. In this sense, BAC has also been shown to induce mitochondrial oxidative stress and detrimental effects on mitochondrial function OSD due to topical preservatives in eye drops is just the tip of the iceberg and the visible consequence of iatrogenic inflammation induced by $\mathrm{BAC}^{9}$.

Lastly, it is important to consider also the effect of BAC on the efficacy of antibiotic therapy. Many studies have shown that the exposure to BAC is linked in promoting antibiotic resistance in environmental bacteria and human pathogenic bacteria ${ }^{10}$.

In conclusion, clinical and experimental evidence demonstrates that BAC causes instability of the tear layer, loss of goblet cells, apoptosis, subclinical neuroinflammation and antibiotic resistance (Figure 1). These findings strongly suggest avoiding the use of this preservative in eye drops. At present, topical formulations containing alternative preservatives (i.e., Polyquad, Purite, SofZia, GenAqua) and preservative-free formulations which exhibit a similar efficacy of eye drop containing BAC with a considerable low grade of ocular toxicity are available. Considering the validity of the alternative formulations, it is unreasonable to persist in using such toxic compounds and, perhaps it is time for a moratorium on the use of BAC in eye drops.

\section{References}

1. Steven DW, Alaghband P, Lim KS. Preservatives in glaucoma medication. The British journal of ophthalmology 2018; 102 (11): 1497-503.

2. Nakamura T, Yamada M, Teshima M, et al. Electrophysiological characterization of tight junctional pathway of rabbit cornea treated with ophthalmic ingredients. Biological $\&$ pharmaceutical bulletin 2007; 30 (12): 2360-4.

3. Pellinen P, Lokkila J. Corneal penetration into rabbit aqueous humor is comparable between preserved and preservative-free tafluprost. Ophthalmic Res 2009; 41 (2): 118-22.

4. Batra R, Tailor R, Mohamed S. Ocular surface disease exacerbated glaucoma: optimizing the ocular surface improves intraocular pressure control. Journal of glaucoma 2014; 23 (1): 56-60.

5. Baudouin C. Ocular Surface and External Filtration Surgery: Mutual Relationships. Developments in ophthalmology 2017; $59: 67-79$.

6. Thygesen J. Glaucoma therapy: preservative-free for all? Clin Ophthalmol 2018; 12 : 707-17.

7. Brignole-Baudouin F, Desbenoit N, Hamm G, et al. A new safety concern for glaucoma treatment demonstrated by mass spectrometry imaging of benzalkonium chloride distribution in the eye, an experimental study in rabbits. PloS one 2012; 7 (11): e50180.

8. Stevens AM, Kestelyn PA, De Bacquer D, Kestelyn PG. Benzalkonium chloride induces anterior chamber inflammation in previously untreated patients with ocular hypertension as measured by flare meter: a randomized clinical trial. Acta Ophthalmol 2012; 90 (3): e221-4.

9. Rogov AG, Goleva TN, Sukhanova EI, et al. Mitochondrial Dysfunctions May Be One of the Major Causative Factors Underlying Detrimental Effects of Benzalkonium Chloride. Oxidative medicine and cellular longevity 2020; 2020 : 8956504.

10. Kim M, Weigand MR, Oh S, et al. Widely Used Benzalkonium Chloride Disinfectants Can Promote Antibiotic Resistance. Applied and environmental microbiology 2018; 84 (17). 


\section{Figure legend}

Figure 1. Detrimental effects of benzalkonium chloride on ocular surface.

\section{Hosted file}

Figure 1..docx available at https://authorea.com/users/458389/articles/554955-is-it-timefor-a-moratorium-on-the-use-of-benzalkonium-chloride-in-eye-drops 